The average distance between the partition and the CRF centre was $5.6 \mathrm{~cm}$ and was always greater than $4 \mathrm{~cm}$. Using results reported previously ${ }^{6}$, we estimated the maximal CRF dimensions as follows. Average CRF centre and surround areas were summed, then doubled. The maximum distance from the centre of the receptive field to the CRF boundary was then estimated as the radius of a circle having this area $(2.4 \mathrm{~cm})$. This conservative estimate is lower than $4 \mathrm{~cm}$. Along with the lack of pyramidal-cell responses to stimulation of the head chamber alone, this indicates that it is very unlikely that the CRF surround extends past the partition.

Received 20 January; accepted 17 March 2003; doi:10.1038/nature01590.

. Rieke, F., Bodnar, D. A. \& Bialek, W. Naturalistic stimuli increase the rate and efficiency of information transmission by primary auditory afferents. Proc. R. Soc. Lond. B 262, 259-265 (1995).

2. Machens, C. K. et al. Representation of acoustic communication signals by insect auditory neurons J. Neurosci. 21, 3215-3227 (2001).

. Nelson, M. E. \& Maclver, M. A. Prey capture in the weakly electric fish Apteronotus leptorhynchus: sensory acquisition strategies and electrosensory consequences. J. Exp. Biol. 202, 1195-1203 (1999).

. Zupanc, G. K. H. \& Maler, L. Evoked chirping in the weakly electric fish Apteronotus leptorhynchus: a quantitative biophysical analysis. Can. J. Zool. 71, 2301-2310 (1993)

Hubel, D. H. \& Wiesel, T. N. Receptive fields, binocular interaction and functional architecture in the cat's visual cortex. J. Physiol. (Lond.) 160, 106-154 (1962).

6. Bastian, J., Chacron, M. J. \& Maler, L. Receptive field organization determines pyramidal cell stimulus-encoding capability and spatial stimulus selectivity. J. Neurosci. 22, 4577-4590 (2002). Sillito, A. M., Grieve, K. L., Jones, H. E., Cudeiro, J. \& Davis, J. Visual cortical mechanisms detecting focal orientation discontinuities. Nature 378, 492-496 (1995).

8. Vinje, W. \& Gallant, J. L. Sparse Coding and decorrelation in primary visual cortex during natural vision. Science 287, 1273-1276 (2000).

9. Simoncelli, E. P. \& Olshausen, B. A. Natural image statistics and neural representation. Annu. Rev. Neurosci. 24, 1193-1216 (2001).

10. Voss, R. F. \& Clarke, J. ' $1 / f$ noise' in music: music from $1 / f$ noise. J. Acoust. Soc. Am. 63, 258-263 (1978).

11. Bastian, J. Electrolocation. I. How the electroreceptors of Apteronotus albifrons code for moving objects and other electrical stimuli. J. Comp. Physiol. A 144, 465-479 (1981).

12. Gabbiani, F., Metzner, W., Wessel, R. \& Koch, C. From stimulus encoding to feature extraction in weakly electric fish. Nature 384, 564-567 (1996).

13. Maler, L., Sas, E. K. \& Rogers, J. The cytology of the posterior lateral line lobe of high frequency weakly electric fish (Gymnotoidei): Dendritic differentiation and synaptic specificity in a simple cortex. J. Comp. Neurol. 195, 87-139 (1981).

14. Rieke, F., Warland, D., de Ruyter van Steveninck, R. R. \& Bialek, W. Spikes: Exploring the Neural Code (MIT, Cambridge, Massachusetts, 1996)

15. Borst, A. \& Theunissen, F. Information theory and neural coding. Nature Neurosci. 2, 947-957 (1999).

16. Mainen, Z. F. \& Sejnowski, T. J. Reliability of spike timing in neocortical neurons. Science $\mathbf{2 6 8}$ 1503-1506 (1995)

17. de Ruyter van Steveninck, R. R., Lewen, G. D., Strong, S. P., Koberle, R. \& Bialek, W. Reproducibility and variability in neural spike trains. Science 275, 1805-1808 (1997)

18. Maler, L. \& Mugnaini, E. Correlating gamma-aminobutyric acidergic circuits and sensory function in the electrosensory lateral line lobe of a gymnotiform fish. J. Comp. Neurol. 345, 224-252 (1994).

19. Berman, N. J. \& Maler, L. Neural architecture of the electrosensory lateral line lobe: Adaptations for coincidence detection, a sensory searchlight and frequency-dependent adaptive filtering. J. Exp. Biol. 202, 1243-1253 (1999)

20. Crampton, W. G. R. Electric signal design and habitat preferences in a species rich assembly of gymnotiform fishes from the upper Amazon basin. Anais Acad. Bras. Cienc. 70, 805-847 (1998).

21. Zhang, H., Xu, J. \& Feng, A. S. Effects of GABA-mediated inhibition on direction-dependent frequency tuning in the frog inferior colliculus. J. Comp. Physiol. 184, 85-98 (1999).

22. Macleod, K. \& Laurent, G. Distinct mechanisms for synchronization and temporal patterning of odor-encoding neural assembies. Science 274, 976-979 (1996).

23. Doiron, B., Chacron, M. J., Maler, L., Longtin, A. \& Bastian, J. Inhibitory feedback required for network burst responses to communication but not prey stimuli. Nature 421, 539-543 (2003).

24. Carr, C. E., Maler, L. \& Sas, E. Peripheral organization and central projections of the electrosensory organs in gymnotiform fish. J. Comp. Neurol. 211, 139-153 (1982).

25. Heiligenberg, W. \& Dye, J. Labelling of electrosensory afferents in a gymnotid fish by intracellular injection of HRP: The mystery of multiple maps. J. Comp. Physiol. A 148, 287-296 (1982).

26. Metzner, W. \& Heiligenberg, W. The coding of signals in the electric communication of the gymnotiform fish Eigenmannia: From electroreceptors to neurons in the torus semicircularis of the midbrain. J. Comp. Physiol. A 169, 135-150 (1991).

27. Metzner, W. \& Juranek, J. A sensory brain map for each behavior? Proc. Natl Acad. Sci. USA 26, 14798-14803 (1997)

28. Heiligenberg, W. Neural Nets in Electric Fish (MIT, Cambridge, Massachusetts, 1991).

29. Bastchelet, E. Circular Statistics in Biology (Academic, New York, 1981).

30. Gabbiani, F. Coding of time varying signals in spike trains of linear and half-wave rectifying neurons. Network Comput. Neural Sys. 7, 61-85 (1996).

Acknowledgements We thank A.-M. Oswald, J. Lewis and B. Lindner for their reading the manuscript. This research was supported by NSERC (M.J.C., B.D., A.L.), CIHR (L.M., A.L.) and NIH (J.B.).

Competing interests statement The authors declare that they have no competing financial interests.

Correspondence and requests for materials should be addressed to M.J.C.

(mchacron@physics.uottawa.ca).

\section{The genome sequence of Bacillus anthracis Ames and comparison to closely related bacteria}

Timothy D. Read ${ }^{\star} \dagger$, Scott N. Peterson ${ }^{\star} \ddagger$, Nicolas Tourasse $\$ \#$, Les W. Baillie ${ }^{\star} \dagger \|$, Ian T. Paulsen ${ }^{\star}$, Karen E. Nelson*, Hervé Tettelin*, Derrick E. Fouts ${ }^{\star}$, Jonathan A. Eisen ${ }^{\star}$, Steven R. Gill ${ }^{*}$, Erik K. Holtzapple ${ }^{\star}$, Ole Andreas $\emptyset k s t a d \$ \#$, Erlendur Helgason $\$ \#$, Jennifer Rilstone*, Martin Wu*, James F. Kolonay*, Maureen J. Beanan*, Robert J. Dodson*, Lauren M. Brinkac ${ }^{*}$, Michelle Gwinn*,

Robert T. DeBoy ${ }^{\star}$, Ramana Madpu*, Sean C. Daugherty* , A. Scott Durkin*, Daniel H. Haft ${ }^{\star}$, William C. Nelson*, Jeremy D. Peterson*, Mihai Pop*, Hoda M. Khouri ${ }^{\star}$, Diana Radune ${ }^{\star}$, Jonathan L. Benton ${ }^{\star}$, Yasmin Mahamoud*, Lingxia Jiang ${ }^{\star}$, loana R. Hance*, Janice F. Weidman*, Kristi J. Berry ${ }^{\star}$, Roger D. Plaut ${ }^{\star}$, Alex M. Wolf*, Kisha L. Watkins ${ }^{\star}$, William C. Nierman ${ }^{\star}$, Alyson Hazen ${ }^{\star}$, Robin Cline ${ }^{\star}$, Caroline Redmond $\dagger$, Joanne E. Thwaite $\dagger$, Owen White ${ }^{\star}$, Steven L. Salzberg ${ }^{\star} \emptyset$, Brendan Thomason $\_$, Arthur M. Friedlander ${ }^{\star *}$, Theresa M. Koehler ††, Philip C. Hanna ${ }^{2}$, Anne-Brit Kolstø\$\# \& Claire M. Fraser ${ }^{\star} \ddagger$ $\$ \subseteq$

* The Institute for Genomic Research, 9712 Medical Center Drive, Rockville, Maryland 20850, USA

$\dagger$ Medical Biotechnology Center, University of Maryland Biotechnology Institute, Baltimore, Maryland 21201, USA

$\ddagger$ Department of Biochemistry, $\ddagger$ Department of Microbiology and Tropical Medicine, $\$ \$$ Department of Pharmacology, The George Washington University, Eye Street, Washington DC 20052, USA

$\$$ School of Pharmacy, University of Oslo N-0316, Oslo, Norway

II Defence Science Technology Laboratory, Porton Down, Salisbury SP4 OJQ, UK

I Johns Hopkins University, Charles and 34th Streets, Baltimore, Maryland 21218, USA

\# The Biotechnology Center of Oslo, Oslo N-0317, Norway

is Department of Microbiology \& Immunology, University of Michigan Medical School, Ann Arbor, Michigan 48109, USA

** US Army Medical Research Institute for Infectious Diseases, Frederick, Maryland 21702, USA

$\dagger \dagger$ Department of Microbiology and Molecular Genetics, University of TexasHouston Health Science Center Medical School, University of Texas, Houston, Texas 77225, USA

Bacillus anthracis is an endospore-forming bacterium that causes inhalational anthrax ${ }^{1}$. Key virulence genes are found on plasmids (extra-chromosomal, circular, double-stranded DNA molecules) pXO1 (ref. 2) and pXO2 (ref. 3). To identify additional genes that might contribute to virulence, we analysed the complete sequence of the chromosome of B. anthracis Ames (about 5.23 megabases). We found several chromosomally encoded proteins that may contribute to pathogenicity-including haemolysins, phospholipases and iron acquisition functions-and identified numerous surface proteins that might be important targets for vaccines and drugs. Almost all these putative chromosomal virulence and surface proteins have homologues in Bacillus cereus, highlighting the similarity of $B$. anthracis to nearneighbours that are not associated with anthrax ${ }^{4}$. By performing a comparative genome hybridization of $19 \mathrm{~B}$. cereus and Bacillus thuringiensis strains against a $B$. anthracis DNA microarray, we confirmed the general similarity of chromosomal genes among this group of close relatives. However, we found that the gene sequences of pXO1 and pXO2 were more variable between strains, suggesting plasmid mobility in the group. The complete sequence of $B$. anthracis is a step towards a better understanding of anthrax pathogenesis.

B. anthracis has become notorious as a bioweapon because of its tough, environmentally resistant endospore and its ability to cause lethal inhalational anthrax. During the course of the disease, 
endospores are taken up by alveolar macrophages where they germinate in the phagolysosomal compartment ${ }^{1}$. Vegetative cells then escape from the macrophage, eventually infecting blood. Expression of the major plasmid-encoded virulence determinants, tripartite toxin and a poly-D-glutamic acid capsule, are essential for full pathogenicity ${ }^{1}$. Sequencing the chromosome of $B$. anthracis was undertaken to help identify additional genes that might contribute to virulence either by encoding functions necessary for the survival and escape from the mammalian macrophage or by enhancing evasion of the immune system and the extent of damage caused by the bacterium to its animal host.

The B. anthracis Ames chromosome sequenced in this work (5,227,293 base pairs, bp) derives from an isolate taken from a dead cow in Texas (Methods). This sequence differs in only 11 confirmed single nucleotide polymorphisms ${ }^{5}$ (SNPs) from the 2001 Florida attack Ames isolate, verifying that the chromosome sequenced to completion is essentially identical to a virulent strain. The chromosome encodes 5,508 predicted protein-coding sequences (Table 1) with a pronounced bias for genes on the replication leading strand (Fig. 1), as has been seen in other low $\mathrm{G}+\mathrm{C}$ Gram-positive replicons ${ }^{6}$. A feature shared with the chromosomes of other endospore-forming Gram-positive species of the genera Bacillus and Clostridium ${ }^{7,8}$ is the concentration of the ribosomal RNA, transfer RNA and ribosomal protein genes around the replication origin. This arrangement may maximize protein synthesis during early rounds of DNA replication after germination from the dormant endospore phase. The chromosome also contains at least four prophages (Supplementary Information) as well as two type I introns, one of which disrupts the recA gene ${ }^{9}$. Housekeeping functions such as DNA replication and fatty-acid metabolism are overwhelmingly partitioned to the chromosome, whereas the pXO1 and $\mathrm{pXO} 2$ plasmids have a greater proportion of transposons, genes involved in toxicity and genes without function assigned (Table 1).

Most B. anthracis Ames chromosomal proteins have homologues to proteins encoded on the draft genome sequence of $B$. cereus ATCC 10987 (T.D.R., unpublished results), a closely related strain (Figs 1 and 2). There are only 141 proteins in B. anthracis for which a putative functional assignment could not be made that do not have a match in the protein set of B. cereus ATCC 10987 sequence (BLASTP ${ }^{10} E<10^{-5}$ ). For the most part, these are encoded by genes of unknown function, are transposases or are present in phage regions. Almost all potential chromosomal virulence-enhancing genes have homologues in B. cereus ATCC 10987, suggesting that they are not specifically associated with the unique pathogenicity of B. anthracis but are part of the common arsenal of the B. cereus group of bacteria ${ }^{11}$.

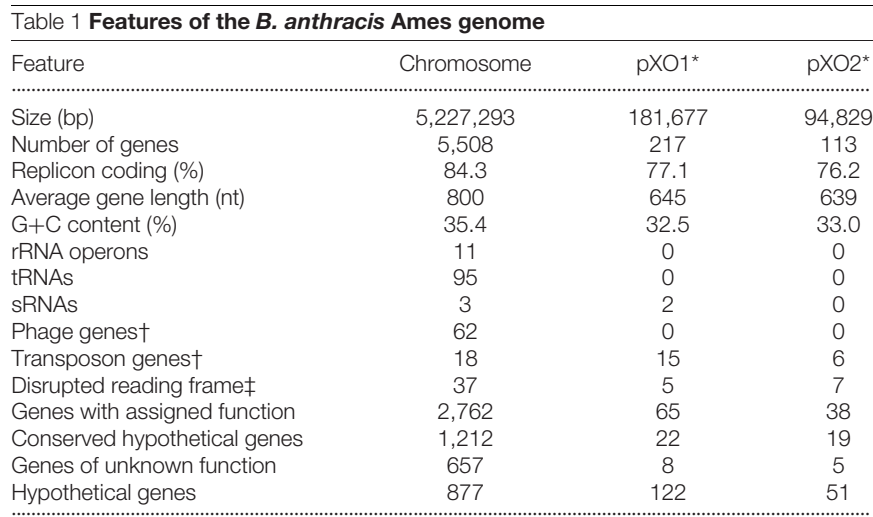

*The complete, annotated pXO1 and pXO2 plasmids from an Ames strain isolated from the 2001 US bioterror attack were also resequenced recently at $\mathrm{TIGR}^{5}$ and have been included in this analysis. †According to TIGR role categories.

$\ddagger$ Genes with indels or point mutations resulting in early termination, confirmed by resequencing.
The chromosome of $B$. anthracis Ames contains several homologues of genes known to be involved in B. cereus and B. thuringiensis pathogenesis. These include two channel-forming type III haemolysins (BA5701, BA2241) and a complex of three non-haemolytic enterotoxins (BA1887-1889). Several B. anthracis Ames proteins have sequence homology to proteins that contribute to the virulence of the Gram-positive pathogen Listeria monocytogenes ${ }^{12}$. These include phosphatidyl-inositol-specific and phosphatidyl-cholinepreferring phospholipase C (BA0677 and BA3891), internalin-like genes (BA1346 and BA1406), listeriolysin O (BA3355), sigma factor B (BA0992) and p60 extracellular protease (BA1952 and BA5474). The significance of these homologies may lie in the similarities in the pathways of intracellular survival and multiplication of L. monocytogenes, and the germination, survival and escape from macrophages by B. anthracis.

$B$. anthracis contains a gene encoding a homologue of the enhancin protein (BA3443), first described in baculoviruses that infect gypsy moths. Enhancin is a metalloprotease that boosts viral infectivity by degrading the mucin layer surrounding insect guts ${ }^{13}$. A homologue of $B$. anthracis enhancin is also found in the genome of Yersinia pestis, which survives in both mammals and insects ${ }^{14}$. $B$. anthracis also contains two homologues of B. thuringiensis immune inhibitor A metalloprotease (BA0672 and BA1295), which enhances virulence in insects through cleavage of bacteriocidal lectins ${ }^{11}$. The presence of these genes may be evidence of an insect-infecting lifestyle in a recent ancestor.

Germination of the anthrax endospore is a key initial event in the B. anthracis infectious cycle. B. anthracis has seven (six chromosomal and one plasmid-borne) paralogues of the gerA family of tricistronic operons utilized by endospores to recognize the presence of specific small molecules to initiate the germination process ${ }^{15}$. Protection of DNA during dormancy and efficient DNA repair during germination are also believed to be important factors in endospore viability. $B$. anthracis has several homologues of the Bacillus subtilis small acid soluble DNA protection proteins, and the full complement of DNA repair proteins found in B. subtilis. B. anthracis also appears to have additional DNA repair capabilities focused on UV-induced DNA damage, with a unique deoxyribodipyrimidine photolyase gene (BA3180) and two, rather than one, UV dimer endonucleases. The photolyase is more closely related to enzymes from proteobacteria than those from other Gram-positive bacteria. The $B$. anthracis genome encodes several proteins that mitigate damage by free-oxygen radicals, including five catalases and three $\mathrm{Fe}-\mathrm{Mn}$ superoxide dismutases. Other detoxification functions for which no obvious homologues could be found in $B$. subtilis include bromoperoxidase, thiolperoxidase, multiple thioredoxin proteins and a cytoplasmic $\mathrm{Cu}-\mathrm{Zn}$ superoxide dismutase (SodC; BA5139). SodC has been shown to have a key role in the virulence of certain other intracellular bacteria, counteracting nitric oxide-mediated killing in the macrophage ${ }^{16}$.

The $B$. anthracis chromosome encodes a machinery for sporulation that is broadly similar to B. subtilis ${ }^{7}$. The proteins with the highest degree of sequence divergence between the species are endospore coat constituents and endospore polysaccharide biosynthesis components, suggesting altered composition of the outer surface. B. subtilis alternative sigma factors, which govern a cascade of events associated with cell development, are also generally conserved. One sigma factor missing in B. anthracis is $s i g D$, which is essential for the expression of the flagellum operon ${ }^{17}$. However, L. monocytogenes, which is motile and carries a flagellum operon similar to $B$. anthracis, also lacks a sigD gene ${ }^{18}$.

Despite having numerous predicted secreted proteins encoded in its genome (Supplementary Information), B. anthracis is notable for paucity of extracellular protease activity under standard laboratory conditions ${ }^{19}$. One reason for this lack of protein secretion may lie in a mutation that affects regulation of gene expression: a nonsense mutation in the plcR positive regulator gene $e^{20}$. In $B$. thuringiensis 
and $B$. cereus, the plcR gene product is known to upregulate the production of numerous extracellular enzymes through binding at

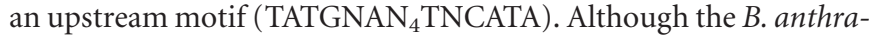
cis plcR homologue is truncated, there are 56 putative plcR binding motifs in the chromosome and 2 on pXO2. The extracellular protein genes downstream include phospholipases, enterotoxins and haemolysins (Table 2), and the plcR mutation has been shown to account for a dramatic reduction in lecithinase, protease and haemolysin production by $B$. anthracis ${ }^{19}$. However, it is possible that some PlcR-regulated gene products still contribute to virulence but are under alternative regulatory controls, as low-level expression of some of the genes in the PlcR regulon has been reported in $B$. anthracis $^{19}$. There is another PlcR-family protein in the genome (BA0597) that might potentially function to complement expression under certain conditions.

The chromosome of $B$. anthracis contains three homologues of the sortase transpeptidase responsible for attachment of secreted proteins to peptidoglycans on the cell surface of Gram-positive bacteria $^{21}$, and also contains the $c s a A B$ genes for binding of proteins with S-layer homology (SLH) domains to polysaccharide. Using searches against models for the sortase attachment sites and SLH domains, 34 candidate surface proteins were identified (Supplementary Information). Two putative B. anthracis sortaseattached genes have internalin-like repeats ${ }^{11}$. The potential role of most proteins with SLH domains on the surface of $B$. anthracis is unknown at present. However, these surface proteins may mediate as-yet-unknown interactions between $B$. anthracis and its external environment, and could be targets for vaccine and drug design.

The broad similarity in metabolic and transport genes of $B$. anthracis and B. subtilis (the model aerobic Gram-positive organism $)^{7}$ suggests many common capabilities, yet there are a number of idiosyncrasies that may shed light on the ecology of B. anthracis. Compared to B. subtilis, B. anthracis appears to have an expanded capacity for amino-acid and peptide utilization. For instance, there are 17 ABC-type peptide binding proteins in

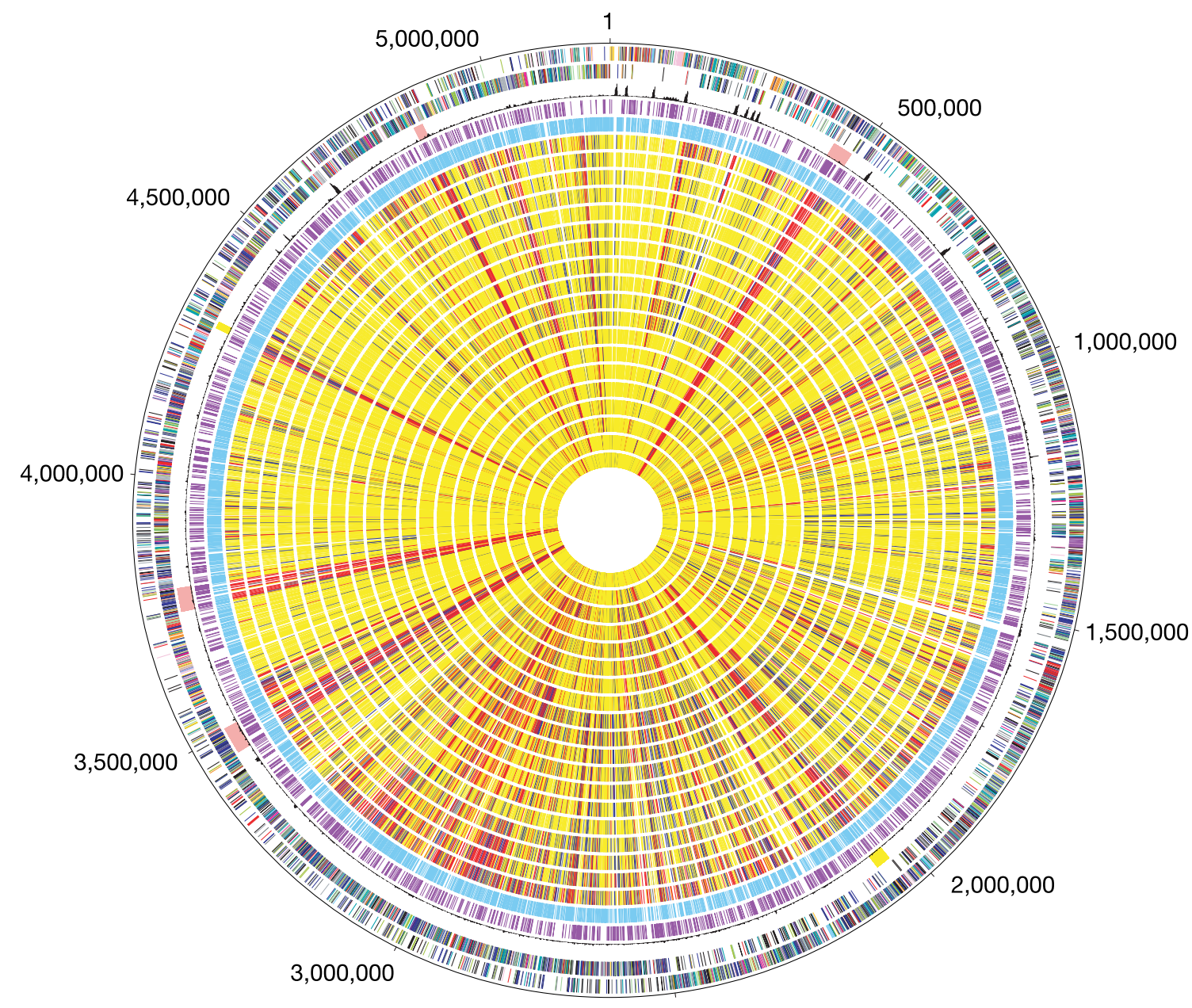

\section{$2,500,000$}

Figure 1 Circular representation of the $B$. anthracis chromosome and comparative genome hybridizations of $B$. cereus group strains. Outer circle, predicted coding regions on the plus strand colour-coded by role categories (see Supplementary Fig. 4). Circle 2 , predicted coding regions on the minus strand colour-coded by role categories. Circle 3 , atypical nucleotide composition curve. Salmon colour, phage regions; yellow, other unique regions located around positions 2.0 and $4.3 \mathrm{Mb}$ (referred to as regions 5 and 6 in the text). Circle 4, genes not represented on the array. Circle 5 , genes present on the array. Genes were classified into three groups: genes present in the query strain (shown yellow), genes absent in the query strain (red), and diverged genes (blue). Missing data are in grey. B. cereus group strains are displayed following the phylogeny of Fig. 2 (circle number, strain number): 6, B.C. $874 ; 7$, B.C. $535 ; 8$, B.c. $612 ; 9$, B.w. 1143; 10, B.t. 248; 11, B.t. 442; 12 , B.c. $14579 ; 13$, B.t. $775 ; 14$, B.c. $259 ; 15$, B.t. $1031 ; 16$, B.t. $251 ; 17$, B.C. $607 ; 18$, B.C. ATCC $10987 ; 19$, B.c. $812 ; 20$, B.c. $819 ; 21$, B.c. $831 ; 22$, B.t. 840 ; 23, B.C. 1123; 24, B.C. 816. Here we use B.C, B.t. and B.w to indicate B. cereus, B. thuringiensis and Bacillus weihenstephanensis, respectively. 
B. anthracis compared with four in B. subtilis; and there are nine homologues of the BrnQ branched chain amino-acid transporter in $B$. anthracis and only two in B. subtilis. B. anthracis also has an expanded number of secreted proteases and peptidases relative to B. subtilis and a number of amino-acid utilization genes not found to date in other Bacillus genomes such as homogentisate dioxygenase (BA0242), involved in tyrosine degradation. Emphasizing the potential importance of peptides and amino acids for $B$. anthracis metabolism, there are six LysE/Rht amino-acid efflux systems compared with two in B. subtilis. These systems prevent accumulation of amino acids to bacteriostatic concentrations during growth on peptides ${ }^{22}$. B. anthracis may therefore be adapted for life in a protein-rich environment, such as decaying animal matter.

$B$. anthracis appears to have a reduced capacity for sugar utilization relative to $B$. subtilis. It lacks catabolic pathways for mannose, arabinose and rhamnose, and has reduced numbers of phosphotransferase systems and other types of sugar transporters. B. anthracis possesses genes for the cleavage of extracellular chitin and chitosan, and the utilization of $\mathrm{N}$-acetylglucosamine constituents of these polymers. This may reflect some type of association with insects analogous to $B$. thuringiensis, or with polymers derived from plant or fungal material. $B$. anthracis contains a complete operon for polyester biosynthesis, which may function as an alternative energy storage compound for the organism. B. anthracis

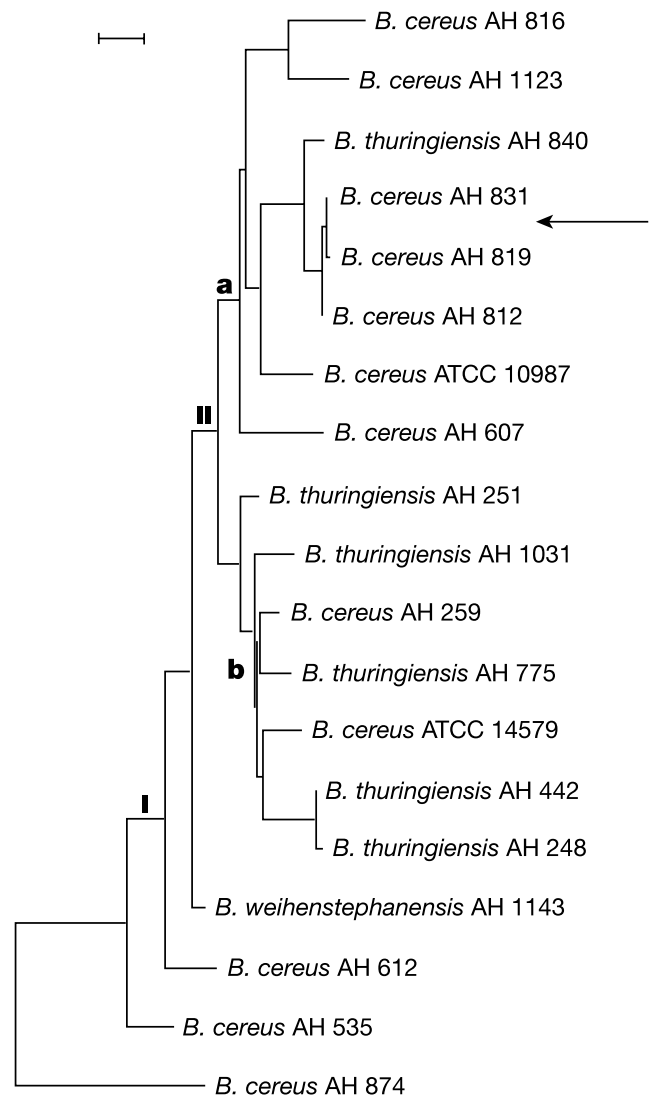

Figure 2 Phylogenetic relationships among 19 B. cereus/B. thuringiensis strains inferred from CGH results for 3,601 chromosomal $B$. anthracis genes. The tree was built by applying the neighbour-joining algorithm to a pairwise distance matrix of percentages of differences between the presence/absence patterns of all strains (diverged genes not taken into account). Similar trees were obtained using the maximum-parsimony method. The scale bar represents $2 \%$ divergence. The arrow indicates the position where $B$. anthracis would emerge by extrapolation from multilocus enzyme electrophoresis analysis ${ }^{4}$. also has a multisubunit NADH hydrogenase not described before in Gram-positive bacteria.

B. anthracis possesses an expanded array of iron-acquisition genes compared to $B$. subtilis that may be important for iron scavenging in a mammalian host. These include 15 ABC uptake systems for iron siderophores or chelates, as well as two clusters of genes for the biosynthesis of siderophores. Two genes involved in synthesis of an aerobactin-like siderophore are not found in $B$. subtilis or the B. cereus ATCC 10987 sequence (BA1981, BA1982). Like B. subtilis and other soil bacteria, B. anthracis encodes a broad swathe of predicted drug efflux pumps, and a variety of other antibiotic-resistance genes are also present. However, it is unknown whether these contribute to resistance in a clinical setting ${ }^{23}$.

We designed a $B$. anthracis DNA microarray on the basis of identifiable genes present at the conclusion of random phase sequencing. The microarray was used to compare $B$. anthracis to 19 members of the $B$. cereus group by comparative genome hybridization (CGH) (Fig. 1). Strains examined by CGH possessed 66-92\% of their chromosomal genes in common with B. anthracis. Genes unique to B. anthracis in particular, and the B. cereus group in general, appear to be over-represented in the $2.0-\mathrm{Mb}$ chromosomal region (coordinates $1,500,000$ to $3,500,000$ in Fig. 1) surrounding the presumed terminus of replication. Genome plasticity around the replication terminus has been seen in other comparisons of bacterial genomes ${ }^{24}$. Six smaller regions of the B. anthracis genome appeared to be absent from nearly all other $B$. cereus group strains tested (Fig. 1). Regions one to four correspond to the B. anthracis prophages (Supplementary Information), and region five centres on an IS110 family insertion element. Only region six does not bear obvious relationship to mobile elements. The magnitude of genomic variability based on CGH experiments comparing the B. anthracis Ames microarray and B. cereus group strains (Fig. 1) is 25-100 times greater than in similar experiments involving comparison of $B$. anthracis Ames to other B. anthracis strains (T. Blank and S.N.P., unpublished results). This reflects the very limited molecular diversity of the B. anthracis species ${ }^{25}$.

Hybridization experiments indicate the presence of $\mathrm{pXO} 1$ homologues in half of the 19 strains examined (Supplementary Information), consistent with what has been shown in other studies $^{26}$. Few genes from the pXO1 pathogenicity island, pXO196 to pXO1-127 (ref. 2), appeared to be present in the 19 B. cereus group strains. The toxin genes, central to anthrax aetiology, are found only in B. anthracis and not in any of the 19 B. cereus group strains sampled. In sharp contrast to pXO1, there were few pXO2 genes hybridizing with genomic DNA from the $19 \mathrm{~B}$. cereus group bacteria.

Ratios obtained by CGH for chromosomal genes were used to infer the phylogenetic relationships among the $B$. cereus strains, producing three clusters (I, IIa and IIb; Fig. 2). The groupings were compatible with the phylogeny reconstructed using multilocus enzyme electrophoresis ${ }^{4}$. By extrapolation of the results from that study to the microarray-based phylogeny, B. anthracis would emerge within cluster IIa (arrow in Fig. 2). The presence of genes with $\mathrm{pXO} 1$ sequence identity in various branches covering all three clusters of the B. cereus group tree (Fig. 2), and the distribution of pXO1-like genes in the $B$. cereus group independent of the chromosomal relatedness among the strains (Supplementary Information), provides further evidence for mobility of pXO1 genes within the $B$. cereus group. Plasmid transfer within the $B$. cereus group is well established ${ }^{27}$, and there are numerous mobility genes on $\mathrm{pXO} 1^{2}$. Despite the evidence for genomic variability in the $B$. cereus group, the $B$. anthracis chromosome and virulence plasmids display little localized variation in $\mathrm{G}+\mathrm{C}$ content and dinucleotide composition (generally associated with horizontally acquired genes from distantly related donors), suggesting that most genes are native to the $B$. cereus group. 
The B. anthracis chromosome sequence portrays a soil-dwelling organism, possessing numerous potential virulence genes, which has possibly a preference for protein-rich environments. This is consistent with the evolution of $B$. anthracis from a $B$. cereus ancestor through acquisition of key plasmid-encoded toxin, capsule and regulatory loci. CGH data presented here demonstrate variability in plasmid gene content among the group as compared to chromosomal genes. Other major differences between $B$. anthracis and $B$. cereus may have been effected through altered gene expression rather than loss or gain of genes. Although both species contain genes associated with secreted proteases, haemolysins, extracellular chitinases ${ }^{11}$, motility, tyrosine degradation and penicillin resistance ${ }^{23}, B$. anthracis and B. cereus phenotypes differ with respect to the function of these genes. These changes in expression

\begin{tabular}{|c|c|c|c|}
\hline \multicolumn{4}{|c|}{ Table 2 Putative PIcR-regulated proteins } \\
\hline Gene & Description & Motif-to-gene distance $(\mathrm{nt})^{\star}$ & Signal peptide $†$ \\
\hline BA0401 & Tellurium resistance protein & 109 & No \\
\hline BA0400 & Tellurium resistance protein & & No \\
\hline BA0399 & Tellurium resistance protein, putative & & No \\
\hline BA0575 & Methyl-accepting chemotaxis protein & 101 & Yes \\
\hline BA0969 & Hypothetical protein & 55 & No \\
\hline BA0975 & HD domain protein & 143 & No \\
\hline BA0976 & Hypothetical protein & 219 & No \\
\hline BA0977 & Hypothetical protein & 157 & Yes \\
\hline BA1086 & Sugar binding transcriptional regulator Lacl family & 73 & No \\
\hline BA1085 & Acetyltransferase, GNAT family & & No \\
\hline BA1424 & Histidyl-tRNA synthetase, putative & 721 & No \\
\hline BA1470 & Membrane protein, putative & 747 & No \\
\hline BA1692 & Conserved hypothetical protein & 171 & No \\
\hline BA1888 & Enterotoxin & 520 & Yes \\
\hline BA1887 & Enterotoxin & & Yes \\
\hline BA1889 & Enterotoxin (point mutation) & & Yes \\
\hline BA2147 & ScdA protein & 16 & No \\
\hline BA2148 & Hypothetical protein & 268 & No \\
\hline BA2499 & Hypothetical protein & 123 & Yes \\
\hline BA2730 & Neutral protease & 114 & Yes \\
\hline BA3355 & Thiol-activated cytolysin & 248 & Yes \\
\hline BA3356 & Membrane protein, putative & 159 & No \\
\hline BA3370 & Ribonuclease & 269 & Yes \\
\hline ВА3491 & Conserved hypothetical protein & 144 & No \\
\hline BA3635 & Spore germination protein & 36 & Yes \\
\hline BA3634 & Spore germination protein & & Yes \\
\hline BA3633 & Spore germination protein & & No \\
\hline BA3891 & 1-Phosphotydlinositol phosphodiesterase & 106 & Yes \\
\hline BA3890 & Hypothetical protein & & No \\
\hline BA3892 & Serine protease, subtilase family & 149 & Yes \\
\hline BA3893 & Cell wall hydrolase, putative & 146 & Yes \\
\hline BA4745 & ABC transporter, ATP-binding protein & 286 & No \\
\hline BA4744 & Membrane protein, putative & & Yes \\
\hline BA4743 & Rrf2 protein family protein, putative & & No \\
\hline BA4746 & Acid phosphatase & 58 & Yes \\
\hline BA4949 & Metallo- $\beta$-lactamase family protein & 84 & No \\
\hline BA5055 & Conserved domain protein & 194 & No \\
\hline BA5190 & Acetyltransferase GNAT family & 93 & No \\
\hline BA5191 & $\mathrm{NAD}(\mathrm{P}) \mathrm{H}$ dehydrogenase, quinone family & 518 & No \\
\hline BA5231 & Hypothetical protein & 14 & No \\
\hline BA5230 & Hypothetical protein & & No \\
\hline BA5243 & CAAX amino terminal protease family protein & 139 & Yes \\
\hline BA5595 & Transcriptional regulator plcR-related, putative, authentic point mutation & 102 & No \\
\hline BA5594 & plcR-associated protein & & Yes \\
\hline BA5596 & Hypothetical protein & 313 & No \\
\hline BA5605 & Hypothetical protein & 113 & No \\
\hline BA5606 & Aminopeptidase, putative & 113 & Yes \\
\hline BA5701 & Channel protein, haemolysin III family & 6 & Yes \\
\hline BA5700 & UDP-galactose-4-epimerase & & No \\
\hline BA5702 & Conserved hypothetical protein & 179 & No \\
\hline
\end{tabular}

†See Supplementary Methods. 
may reflect recent adaptations following acquisition of the pathogenicity island that contains the lethal toxin loci on $\mathrm{pXO1}$. The atxA regulatory gene in this region controls toxin gene expression but is incompatible with the chromosomal regulator $p l c R$, found in B. cereus $^{19}$. The worldwide, near-clonal spread of the organism ${ }^{25}$ suggests that expression of the toxin and capsule genes confers an advantage to $B$. anthracis that outweighs changes in the chromosomal gene expression. Findings from this genome sequence analysis raise further questions about the biology of $B$. anthracis; for instance, what are the roles of putative 'virulence' genes in close relatives of $B$. anthracis that do not cause anthrax, and do they actually contribute to virulence in $B$. anthracis?

\section{Methods}

Genome sequencing and analysis of $B$. anthracis Ames (pX01- $\mathrm{pXO2}^{-}$)

B. anthracis Ames was cured of plasmid pXO1 by incubation at $43^{\circ} \mathrm{C}$ and $\mathrm{pXO} 2$ subsequently cured by novobiocin treatment (Supplementary Methods). As previously described, the chromosome was sequenced using two DNA preparations. For the first $(\text { Porton } 1)^{5}, 2-3$ kilobase $(\mathrm{kb})$ and $4-7 \mathrm{~kb}$ random insert libraries in plasmid-derived vectors were constructed and end-sequenced following the standard strategy for TIGR microbial shotgun projects 6 , achieving success rates of $74 \%$ and $64 \%$ and average highquality read lengths of 559 nucleotides (nt) and $586 \mathrm{nt}$, respectively. For the Porton2 preparation ${ }^{5}$, libraries of $2-3 \mathrm{~kb}$ and $6-8 \mathrm{~kb}$ were constructed with success rates of $89 \%$ and $85 \%$ and average high-quality read lengths of $609 \mathrm{nt}$ and $645 \mathrm{nt}$. The completed chromosome sequence consisted of 73,806 and 6,052 reads from the Porton1 small and large insert libraries, and 3,532 and 32,430 from the Porton2 small and large insert libraries-achieving an average of 13 -fold sequence coverage per base. After assembly, gaps between contigs were closed by editing, walking library clones, and linking assemblies by polymerase chain reaction (PCR). The Glimmer gene finder ${ }^{28}$ was modified by enhancing its model of noncoding sequences. This improved its ability to exclude short open reading frames (ORFs), and substantially reduced the number of predicted small hypothetical proteins. Annotation was as described for a previous project ${ }^{6}$. BLASTP ${ }^{10}$ was used for comparisons of the protein sets of B. anthracis, B. cereus ATCC 10987 (T.D.R., unpublished results; http://www.tigr.org/tdb/ufmg/) and other complete bacterial genomes (http://www.tigr.org/cmr2/ and http://genolist.pasteur.fr/ Subtilist/). A predicted probability score of less than $10^{-5}$ was used as a standard cut-off to define a likely match.

\section{DNA microarray preparation and analysis}

Amplicons representing 79 of 217 and 41 of 122 genes from $\mathrm{pXO} 1$ and $\mathrm{pXO} 2$ respectively, and 3,601 of 5,753 chromosomal genes as predicted by Glimmer ${ }^{28}$ (see Supplementary Methods) were arrayed onto glass microscope slides (Telechem Inc.). Redundant genes were generally represented once or a few times on the array. Genomic DNA was labelled with Cy3 and Cy5 according to J. DeRisi (http://www.microarrays.org/Pdfs/ GenomicDNALabel_B.pdf), except that genomic DNA was not digested or sheared before labelling. Arrays were scanned with a GenePix 4000B scanner (Axon Inc.). Hybridization signals were quantified using TIGR SPOTFINDER (software available at http:// www.tigr.org/softlab). Hybridization experiments were competitive using probes derived from B. anthracis Ames (reference) and a B. cereus group (query) strain. Normalized signal intensities were used to generate relative hybridization ratios (query/reference). Data representing weak signal were removed. The ratios from a maximum of six data points (duplicate spots, hybridizations performed in triplicate) were placed in three bins: $<0.1$, gene is absent in query strain; $0.1-0.3$, present but diverged in query strain; and $>0.3$, gene is present in the query strain. A majority rule was applied to the data for binning such that more than $50 \%$ of ratios were in agreement as to assignment and that at least two data points were used (exceeded in $99 \%$ of the cases). In cases where less than two data points existed, the gene was treated as data missing.

The criteria for the numerical ranges of our bins were established in two ways. First, we determined the presence or absence of sequences homologous to 3,601 B. anthraci genes in the sequence of B. cereus ATCC 14579 (Integrated Genomics Inc.; http:// www.integratedgenomics.com/) using BLASTN ${ }^{10}$, and compared that to the assignments inferred from hybridization ratios. A threshold of 0.1 was found to be suitable for classifying a gene as absent (that is, agreement between sequence and CGH data in 99\% of the cases), while a cut-off value of 0.3 was conservative for gene presence (agreement in $92 \%$ of the cases). Second, we used a set of 65 genes conserved in 26 bacterial genomes, NCBI COG database (http://www.ncbi.nlm.nih.gov/COG/). Genes judged as present in query strains using our selected cut-offs correctly binned data in 1,225 out of 1,235 total calls. There was a tendency for underprediction of plasmid homologues by CGH, when compared to results from the sequence analysis. Two possible explanations for this are variability in plasmid copy number in $B$. cereus strains relative to $B$. anthracisl and/or that the average divergence of plasmid genes is greater than chromosomal genes.

\section{Other techniques and analysis}

PCR amplification for microarray spotting, pulsed field gel electrophoresis and Southern blotting are described in Supplementary Information, as is the phylogenetic analysis of chromosomal data.
Received 14 August 2002; accepted 28 March 2003; doi:10.1038/nature01586.

1. Dixon, T. C., Meselson, M., Guillemin, J. \& Hanna, P. C. Anthrax. N. Engl. J. Med. 341, 815-826 (1999).

2. Okinaka, R. T. et al. Sequence and organization of $\mathrm{pXO1}$, the large Bacillus anthracis plasmid harboring the anthrax toxin genes. J. Bacteriol. 181, 6509-6515 (1999).

3. Okinaka, R. et al. Sequence, assembly and analysis of $\mathrm{pXO1}$ and pXO2. J. Appl. Microbiol. 87, 261-262 (1999).

4. Helgason, E. et al. Bacillus anthracis, Bacillus cereus, and Bacillus thuringiensis-one species on the basis of genetic evidence. Appl. Environ. Microbiol. 66, 2627-2630 (2000).

5. Read, T. D. et al. Comparative genome sequencing for discovery of novel polymorphisms in Bacillus anthracis. Science 296, 2028-2033 (2002).

6. Tettelin, H. et al. Complete genome sequence of a virulent isolate of Streptococcus pneumoniae. Science 293, 498-506 (2001).

7. Kunst, F. et al. The complete genome sequence of the gram-positive bacterium Bacillus subtilis. Nature 390, 249-256 (1997).

8. Nolling, J. et al. Genome sequence and comparative analysis of the solvent-producing bacterium Clostridium acetobutylicum. J. Bacteriol. 183, 4823-4838 (2001).

9. Ko, M., Choi, H. \& Park, C. Group I self-splicing intron in the recA gene of Bacillus anthracis. J. Bacteriol. 184, 3917-3922 (2002).

10. Altschul, S. F. et al. Gapped BLAST and PSI-BLAST: a new generation of protein database search programs. Nucleic Acids Res. 25, 3389-3402 (1997).

11. Guttmann, D. M. \& Ellar, D. J. Phenotypic and genotypic comparisons of 23 strains from the Bacillus cereus complex for a selection of known and putative B. thuringiensis virulence factors. FEMS Microbiol. Lett. 188, 7-13 (2000).

12. Cossart, P. Molecular and cellular basis of the infection by Listeria monocytogenes: an overview. Int. J. Med. Microbiol. 291, 401-409 (2002).

13. Lepore, L. S., Roelvink, P. R. \& Granados, R. R. Enhancin, the granulosis virus protein that facilitates nucleopolyhedrovirus (NPV) infections, is a metalloprotease. J. Invertebr. Pathol. 68, 131-140 (1996).

14. Parkhill, J. et al. Genome sequence of Yersinia pestis, the causative agent of plague. Nature 413, $523-527$ (2001).

15. McCann, K. P., Robinson, C., Sammons, R. L., Smith, D. A. \& Corfe, B. M. Alanine germination receptors of Bacillus subtilis. Lett. Appl. Microbiol. 23, 290-294 (1996).

16. De Groote, M. A. et al. Periplasmic superoxide dismutase protects Salmonella from products of phagocyte NADPH-oxidase and nitric oxide synthase. Proc. Natl Acad. Sci. USA 94, 13997-14001 (1997)

17. West, J. T., Estacio, W. \& Marquez-Magana, L. Relative roles of the fla/che $\mathrm{P}(\mathrm{A}), \mathrm{P}(\mathrm{D}-3)$, and $\mathrm{P}($ sigD $)$ promoters in regulating motility and sigD expression in Bacillus subtilis. J. Bacteriol. 182, 4841-4848 (2000)

18. Glaser, P. et al. Comparative genomics of Listeria species. Science 294, 849-852 (2001).

19. Mignot, T. et al. The incompatibility between the PlcR- and AtxA-controlled regulons may have selected a nonsense mutation in Bacillus anthracis. Mol. Microbiol. 42, 1189-1198 (2001).

20. Agaisse, H., Gominet, M., Okstad, O. A., Kolsto, A. B. \& Lereclus, D. PlcR is a pleiotropic regulator of extracellular virulence factor gene expression in Bacillus thuringiensis. Mol. Microbiol. 32, 1043-1053 (1999).

21. Pallen, M. J., Lam, A. C., Antonio, M. \& Dunbar, K. An embarrassment of sortases-a richness of substrates? Trends Microbiol. 9, 97-102 (2001).

22. Bellmann, A. et al. Expression control and specificity of the basic amino acid exporter LysE of Corynebacterium glutamicum. Microbiology 147, 1765-1774 (2001).

23. Turnbull, P. C. Definitive identification of Bacillus anthracis-a review. J. Appl. Microbiol. 87, 237-240 (1999).

24. Suyama, M. \& Bork, P. Evolution of prokaryotic gene order: genome rearrangements in closely related species. Trends Genet. 17, 10-13 (2001).

25. Keim, P. et al. Multiple-locus variable-number tandem repeat analysis reveals genetic relationships within Bacillus anthracis. J. Bacteriol. 182, 2928-2936 (2000).

26. Pannucci, J., Okinaka, R. T., Sabin, R. \& Kuske, C. R. Bacillus anthracis pXO1 plasmid sequence conservation among closely related bacterial species. J. Bacteriol. 184, 134-141 (2002).

27. Thorne, C. B. Bacillus subtilis and Other Gram-positive Bacteria 113-124 (American Society for Microbiology, Washington DC, 1993).

28. Salzberg, S. L., Delcher, A. L., Kasif, S. \& White, O. Microbial gene identification using interpolated Markov models. Nucleic Acids Res. 26, 544-548 (1998).

Supplementary Information accompanies the paper on www.nature.com/nature.

Acknowledgements We acknowledge the contributions of P. Turnbull, E. Saile, Y. Chen, J. Hunter-Cevera, N. McKinney, S. Cendrowski, M. Weiner, A. Fouet, A. Harrison, S. Leppla, M. Mock, C. Moran, G. Myers, G. Patra, J. Ravel, E. Reilly and T. Torok. The B. anthracis chromosome sequence was supported by funding from the Office of Naval Research (ONR), National Institutes of Allergy and Infectious Disease (NIAID), the Department of Energy and the UK Defence Science Technology Laboratory. Comparative genome hybridization experiments were supported by the ONR. A-B.K, N.T, O.A.O. and E.H were supported by the Norwegian Research Council. The sequencing of B. cereus ATCC 10987 was supported by the NIAID under the Pathogen Functional Genomics Resource Center contract, and the ONR.

Competing interests statement The authors declare that they have no competing financial interests.

Correspondence and requests for materials should be addressed to T.D.R. (anthrax@tigr.org). The B. anthracis genome sequence has been deposited at GenBank under accession number AE016879; the microarray data have been deposited at Gene Expression Omnibus (GEO) under accession number GSE341. The B. cereus 10987 unfinished genome sequence (GenBank accession number NC_003909) is available at http://www.tigr.org/tdb/ufmg). 